\title{
Basal-Bolus Insulin Therapy with Gla-300 During Hospitalization Reduces Nocturnal Hypoglycemia in Patients with Type 2 Diabetes Mellitus: A Randomized Controlled Study
}

\author{
Fumitaka Okajima (D) Y Yko Nakamura - Yuji Yamaguchi · \\ Yuki Shuto $\cdot$ Katsuhito Kato $\cdot$ Hitoshi Sugihara $\cdot$ Naoya Emoto
}

Received: November 6, 2017 / Published online: April 4, 2018

(c) The Author(s) 2018

\section{ABSTRACT}

Introduction: Although reduction in the incidence of nocturnal hypoglycemia, as estimated by symptom or self-monitored plasma glucose, was shown to be more pronounced with 300 units/mL insulin glargine (Gla-300) than with 100 units/mL insulin glargine (Gla-100) in type 2 diabetes patients, the exact frequency of nocturnal hypoglycemia estimated with continuous glucose monitoring (CGM) has not been reported.

Methods: Forty patients with type 2 diabetes who were admitted for glycemic control with basal-bolus insulin therapy (BBT) were randomized into the Gla-100 and Gla-300 groups.

Enhanced content To view enhanced content for this article go to https://doi.org/10.6084/m9.figshare. 6016238.

F. Okajima ( $₫)$. Y. Nakamura · Y. Yamaguchi ·

Y. Shuto $\cdot$ N. Emoto

Department of Endocrinology, Chiba-Hokusoh Hospital, Nippon Medical School, Inzai, Chiba, Japan

e-mail: okaji@nms.ac.jp

F. Okajima · Y. Nakamura · Y. Yamaguchi ·

Y. Shuto $\cdot$ H. Sugihara · N. Emoto

Department of Endocrinology, Diabetes and

Metabolism, Graduate School of Medicine, Nippon

Medical School, Tokyo, Japan

K. Kato

Department of Hygiene and Public Health, Nippon

Medical School, Tokyo, Japan
Insulin doses were adjusted to maintain blood glucose levels within 100-120 mg/dL at each meal. Plasma glucose and C-peptide profiles were estimated serially after admission and before discharge. Daily CGM was also performed before discharge.

Results: In the Gla-100 and Gla-300 groups, the mean duration of hospitalization was $15 \pm 2$ and $15 \pm 1$ days, respectively, and the mean basal insulin dose before discharge was $13 \pm 7$ and $15 \pm 10$ units, respectively. The dose of meal-time insulin was not different between the two groups. Compared with the Gla-300 group, the Gla-100 group had significantly lower nocturnal profiles of plasma glucose and C-peptide, but significantly higher frequency of CGM-estimated nocturnal hypoglycemia $(10.7 \% \pm$ $18.4 \%$ versus $1.2 \% \pm 3.6 \%, P=0.033$ ).

Conclusion: In type 2 diabetic patients, reduction in the incidence of CGM-estimated nocturnal hypoglycemia by BBT under tightly controlled diet therapy was higher with Gla-300 than with Gla-100.

Trial Registration: UMIN clinical trials registry (UMIN000023360).

Keywords: Basal-bolus insulin therapy; Continuous glucose monitoring; Insulin glargine $300 \mathrm{U} / \mathrm{mL}$; Nocturnal hypoglycemia; Type 2 diabetes mellitus 


\section{INTRODUCTION}

Basal-bolus insulin therapy (BBT) is the most effective approach to ameliorate hyperglycemia, but has adverse effects, such as weight gain and hypoglycemia, which may increase the incidence of cardiovascular events. During the daytime, a patient can become aware of the symptoms of hypoglycemia, such as palpitations, cold sweats, etc. However, nocturnal hypoglycemia is difficult to notice for a patient. With continuous glucose monitoring (CGM), nocturnal hypoglycemia, including asymptomatic hypoglycemia, can be detected with high precision [1]. Because of a flatter pharmacokinetics (PK) scope, therapy with insulin glargine 100 units/mL (Gla-100) was reported to significantly reduce the incidence of severe symptomatic nocturnal hypoglycemia, compared with therapy with neutral protamine Hagedorn insulin [2]. However, our previous investigation on hospitalized type 2 diabetic patients with tightly controlled blood sugar under BBT showed that Gla-100 induced asymptomatic hypoglycemia, confirmed by CGM, in high frequency [3].

On the other hand, insulin glargine 300 units/mL (Gla-300) is a new basal insulin analogue that has a prolonged effect and flatter PK profile than Gla-100 [4-6]. Previous studies on type 2 diabetic patients treated with BBT, basal supported oral therapy, or oral antidiabetic agents alone showed that compared with Gla-100, Gla-300 could control HbA1c at the same efficacy and with consistently less risk of nocturnal hypoglycemia at 3:00, as estimated by symptoms and self-monitored plasma glucose [7-12]. Two studies that employed CGM demonstrated the safety and efficacy of Gla-300 in type 1 diabetics $[13,14]$; however, research on CGM in type 2 diabetic patients has not been reported.

In the present study, we evaluated by CGM the efficacy and safety of BBT with Gla-300 in comparison with that with Gla-100 in wellcontrolled type 2 diabetic patients at the end of short-term hospitalization.

\section{METHODS}

\section{Subjects}

In the present study, we included the type 2 diabetes patients, who consulted the outpatient clinic of Nippon Medical School Chiba Hokusoh Hospital from November 2014 to October 2016 if they had HbA1c level $\geq 10 \%$ on the first visit and had consented for hospitalization for diabetic control. The hospital is one of the three base hospitals in a locality with a population of 700,000 that consists of several cities, towns, and villages near the metropolitan city of Tokyo, Japan. The clinic provides care to patients with diabetes referred by general practitioners or other departments in the hospital because the diabetes was difficult to control because of poor metabolic control or unstable complications. The socioeconomic status of the patients was previously reported [15]. Subjects were excluded if they had been previously treated with insulin; were positive for anti-glutamic acid decarboxylase antibody; or had history or evidence of recent myocardial infarction, heart failure, cerebrovascular disease, endocrine disease, or any carcinoma.

A total of 54 patients with type 2 diabetes were assessed for eligibility. Patients were excluded because of the presence of concomitant disease $(n=7)$, insulin use $(n=3)$, and rejection of approval $(n=4)$. The remaining 40 patients, aged 20-75 years, were enrolled in an unblinded, randomized study (Fig. 1).

\section{Study Protocol and Treatment}

The study protocol was approved by the ethics committee of Nippon Medical School Chiba Hokusoh Hospital (No.527009) and was registered at UMIN Clinical Trials Registry (UMIN000023360). All procedures followed were in accordance with the ethical standards of the responsible committee on human experimentation (institutional and national) and with the Helsinki Declaration of 1964, as revised in 2013. Informed consent was obtained from all patients for inclusion in the study. On admission, all subjects stopped taking oral 


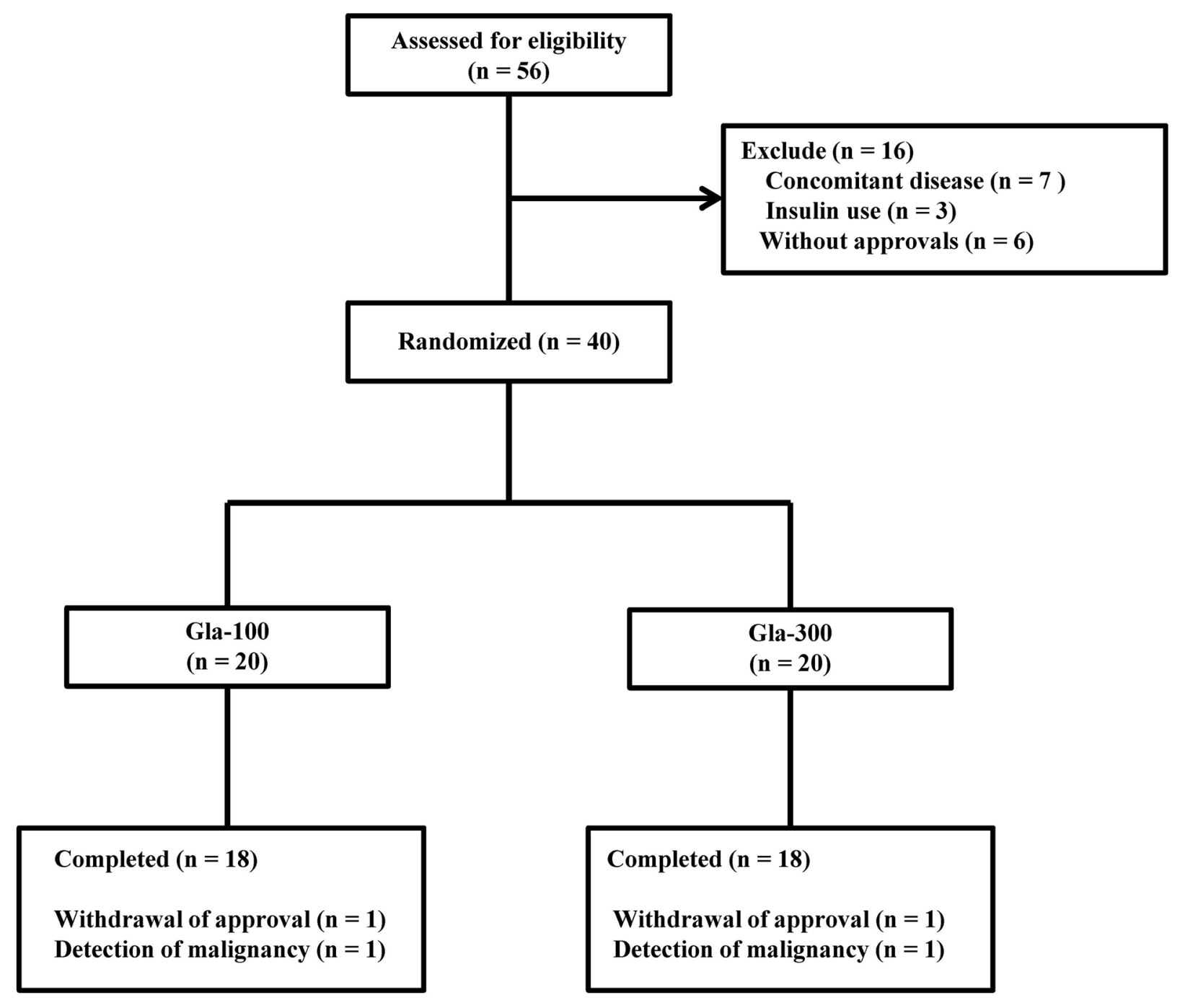

Fig. 1 Participant flow

antidiabetic agents, received diet therapy, and were randomly assigned using a table of random sampling numbers (block size of 4) to receive BBT with insulin glulisine plus either Gla-100 (Gla-100 group, $n=20$ ) or Gla-300 (Gla-300 group, $n=20$ ).

In both groups, the dose of insulin injection was adjusted by the attending physicians to maintain pre-meal blood glucose levels within $100-120 \mathrm{mg} / \mathrm{dL}$. Within 3 days after admission, an ophthalmologist checked each patient for the presence of diabetic retinopathy; if needed, fluorescent fundus angiography and retinal laser photocoagulation were immediately performed.
To assess the daily profile of plasma glucose and C-peptide (CPR), blood specimens were obtained from the cubital vein before and every $2 \mathrm{~h}$ after each meal (8:00, 10:00, 12:00, 14:00, 18:00, and 20:00); at night (23:00); in the early morning (3:00); and on the next morning (8:00). These processes were performed on the first and second days after admission and on the last 2 days before discharge. Analysis of daily profile was performed for the whole day; during the daytime including 8:00, 10:00, 12:00, 14:00, 18:00, and 20:00; and at night including 23:00, 3:00, and 8:00 of the next morning. The blood samples collected between 7:30 and 14:00 were immediately centrifuged, whereas those 
collected between 17:00 and 3:00 were stored at $4^{\circ} \mathrm{C}$ and centrifuged the next morning.

Daily blood glucose profiles on the last 2 days before discharge were assessed by CGM (iPro ${ }^{\mathrm{TM}} 2$; Medtronic, Minneapolis, MN, USA). To assess daily glycemic variability, the mean glucose level, standard deviation (SD) of the daily glucose, and mean amplitude of glycemic excursion (MAGE) [16] were calculated. Hypoglycemia was defined as a CGM value of lower than $70 \mathrm{mg} / \mathrm{dL}$. A hypoglycemic episode that occurred anytime from 0:00 to 8:00 was defined as nocturnal hypoglycemia.

\section{Statistical Analysis}

All analyses except two-way repeated measures analysis of variance were performed using the JMP 12.2 software (SAS Institute, Cary, NC). Values were presented as mean \pm SD or number. Statistical analyses of the gender differences and complications of diabetes at baseline were performed using the $\chi^{2}$ test. The significance of the differences between two groups with regard to the baseline characteristics and parameters of glycemic control before discharge was analyzed by Student's $t$ test. Two-way repeated measures analysis of variance of the daily profile of PG and CPR were performed using IBM SPSS
Statistics software (version 22; IBM Japan, Tokyo, Japan). A $P$ value of less than 0.05 was considered statistically significant.

\section{RESULTS}

The present study enrolled 40 patients, with mean age of $58 \pm 14$ years, who had type 2 diabetes for a mean duration of $5 \pm 6$ years; $72.5 \%$ were men (Table 1 ). Four patients, two from each group, dropped out because of the detection of malignancy or withdrawal of approval (Fig. 1). There were no significant differences in the baseline characteristics between the two treatment groups.

The duration of hospitalization was $15 \pm 2$ and $15 \pm 1$ days in the Gla-100 and Gla-300 groups, respectively. The required insulin doses to maintain normoglycemia were not different between the two groups; specifically, the required doses for Gla-100 and Gla-300 were $10 \pm 5$ and $10 \pm 6$ units, respectively, before breakfast; $3 \pm 2$ and $2 \pm 2$ units, respectively, before lunch; $9 \pm 3$ and $9 \pm 4$ units, respectively, before dinner; and $13 \pm 7$ and $15 \pm 10$ units, respectively, at bedtime.

The daily profiles of plasma glucose and CPR were not significantly different between the two groups upon admission, but were significantly

Table 1 Baseline parameters of glycemic control, complications, and medications before admission

\begin{tabular}{llll}
\hline Parameter & Gla-100 & Gla-300 & P value \\
\hline Number of patients (men) & $20(13)$ & $20(16)$ & NS \\
Age (years) & $61 \pm 12$ & $54 \pm 15$ & NS \\
Duration of diabetes (years)* & $6 \pm 7$ & $5 \pm 6$ & NS \\
BMI $\left(\mathrm{kg} / \mathrm{m}^{2}\right)$ & $26.1 \pm 3.9$ & $25.5 \pm 6.3$ & NS \\
Designated dietary calorie (kcal/day) & $1594 \pm 231$ & $1661 \pm 217$ & NS \\
FPG $(\mathrm{mg} / \mathrm{dL})$ & $198 \pm 54$ & $199 \pm 51$ & NS \\
HbAlc $(\%)^{* *}$ & $11.1 \pm 2.1$ & $11.0 \pm 2.2$ & NS \\
GA $(\%)$ & $27 \pm 6.8$ & $28.6 \pm 9.9$ & NS \\
ALT $(\mathrm{mg} / \mathrm{dL})$ & $29 \pm 17$ & $23 \pm 9$ & NS \\
AST $(\mathrm{mg} / \mathrm{dL})$ & $30 \pm 21$ & $26 \pm 13$ & NS \\
GGT $(\mathrm{mg} / \mathrm{dL})$ & $57 \pm 72$ & $43 \pm 33$ & NS \\
\hline
\end{tabular}


Table 1 continued

\begin{tabular}{|c|c|c|c|}
\hline Parameter & Gla-100 & Gla-300 & $P$ value \\
\hline Cre $(\mathrm{mg} / \mathrm{dL})$ & $0.71 \pm 0.21$ & $0.73 \pm 0.29$ & NS \\
\hline U-CPR ( $\mu \mathrm{g} /$ day $)$ & $116 \pm 67.5$ & $133 \pm 65$ & NS \\
\hline \multicolumn{4}{|l|}{ Complication } \\
\hline Absent Achilles tendon reflex $(n)$ & 9 & 10 & NS \\
\hline U-Alb (mg/day) & $94.4 \pm 188$ & $311.2 \pm 865.2$ & NS \\
\hline Diabetic retinopathy $(\mathrm{DR})^{* * *}$ & & & NS \\
\hline None $(n)$ & 14 & 11 & \\
\hline Simple DR $(n)$ & 5 & 8 & \\
\hline Preproliferative DR $(n)$ & 1 & 1 & \\
\hline Proliferative DR $(n)$ & 0 & 0 & \\
\hline Medication before admission* & & & NS \\
\hline Sulfonylureas $(n)$ & 4 & 4 & \\
\hline Biguanides $(n)$ & 3 & 4 & \\
\hline Thiazolidinediones $(n)$ & 1 & 0 & \\
\hline Alpha-glucosidase inhibitors $(n)$ & 3 & 1 & \\
\hline Dipeptidyl peptidase- 4 inhibitor $(n)$ & 5 & 4 & \\
\hline
\end{tabular}

Data are expressed as mean \pm standard deviation or number

Gla-100 insulin glargine 100 units/mL, Gla-300 insulin glargine 300 units/mL, NS not significant, BMI body mass index, FPG fasting plasma glucose, $H b A 1 c$ hemoglobin Alc, $G A$ glycated albumin, $A S T$ aspartate aminotransferase, $A L T$ alanine aminotransferase, $G G T$ gamma glutamyl transpeptidase, Cre creatinine, $U$ - $C P R$ urinary C-peptide immunoreactivity, $U$ - $A l b$ urinary albumin

* Reported by the general practitioners who referred the patients

**HbA1c levels were determined by high-performance liquid chromatography using an HLC723G8 (Tosoh, Co., Tokyo, Japan) and presented as the equivalent National Glycohemoglobin Standardization Program values

***Evaluated by ophthalmologists who performed fundoscopy after pupillary dilatation following the Davis classification

lower in the Gla-100 group than in the Gla-300 group only at night before discharge (Fig. 2 and Table 2$)$. The mean glucose $(119 \pm 15$ and $124 \pm 20$ in Gla-100 and Gla-300 group, respectively), $\mathrm{SD}(33 \pm 11$ and $31 \pm 12)$, and MAGE $(79 \pm 36$ and $74 \pm 27)$ levels calculated with CGM data before discharge were not significantly different between the BBT groups. However, in both groups, the nadir of the blood glucose profile was around 0:00-1:00 a.m. (Fig. 3). The frequency of nocturnal hypoglycemia was significantly lower in the Gla-300 group than in the Gla-100 group $(1.2 \% \pm 3.6 \%$ versus $10.7 \% \pm 18.4 \%, P=0.039$ ) (Figs. 3 and
4). No patients reported nocturnal hypoglycemic symptoms, such as night sweats and nightmares, during the admission.

No adverse events, such as symptomatic hypoglycemia, injection-site reaction, infection gastrointestinal events, musculoskeletal complaints, or newly developed blood test abnormality during admission, were reported.

\section{DISCUSSION}

The present study, which employed CGM and serial phlebotomies to compare Gla-300 and 

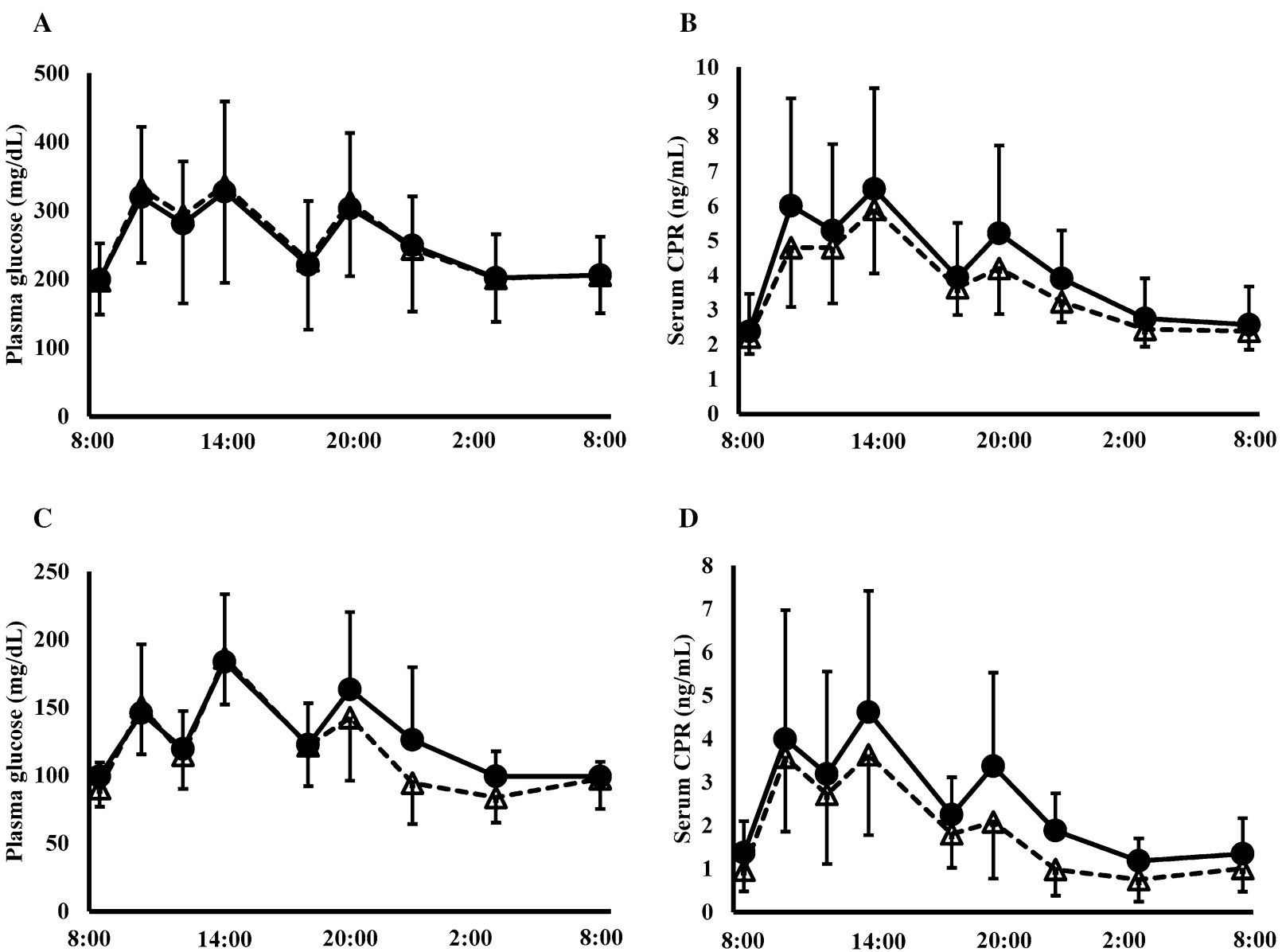

D

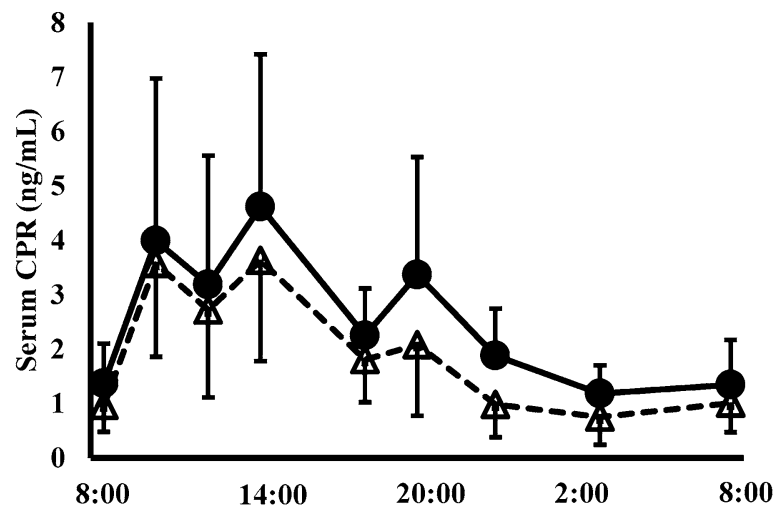

Fig. 2 Daily profiles of plasma glucose and serum C-peptide. Daily profiles of plasma glucose $(\mathbf{a}, \mathbf{c})$ and serum C-peptide (b, d) in the insulin glargine 100 units $/ \mathrm{mL}$ group (open triangles and dashed line; $n=18$ ) and in the

Gla-100, revealed no difference in the required dose of insulin and time to achieve normoglycemia. However, the frequency of nocturnal hypoglycemia was significantly lower with Gla300 than with Gla-100. In similar studies, $[7-9,11,12]$ the basal insulin dose required to achieve the same fasting plasma glucose had a non-significant and slight tendency to be higher with Gla-300 than with Gla-100. The required dose of prandial insulin was not different between the two groups.

The higher required dose of Gla-300 probably reflects the slightly lower serum glargine concentration at $11 \mathrm{~h}$ after administration, which could be explained by some PK-pharmacodynamics (PD) studies that showed

reduced absorption of the more concentrated Gla-300 insulin in subcutaneous administration, even at equal doses with Gla-100 [4-6].

In the present study, the nocturnal plasma glucose and CPR levels estimated by serial phlebotomies were significantly lower in Gla100 than in Gla-300, probably owing to the stronger glucose-lowering effect of the infused Gla-100 in the nocturnal phase. The CGM-estimated nocturnal glucose levels in the Gla-100 group were lower in the hypoglycemic range at a high frequency than those in the Gla-300 group.

The reduction of frequency in the nocturnal hypoglycemia in type 2 diabetic patients in this study was the same as the results shown in 


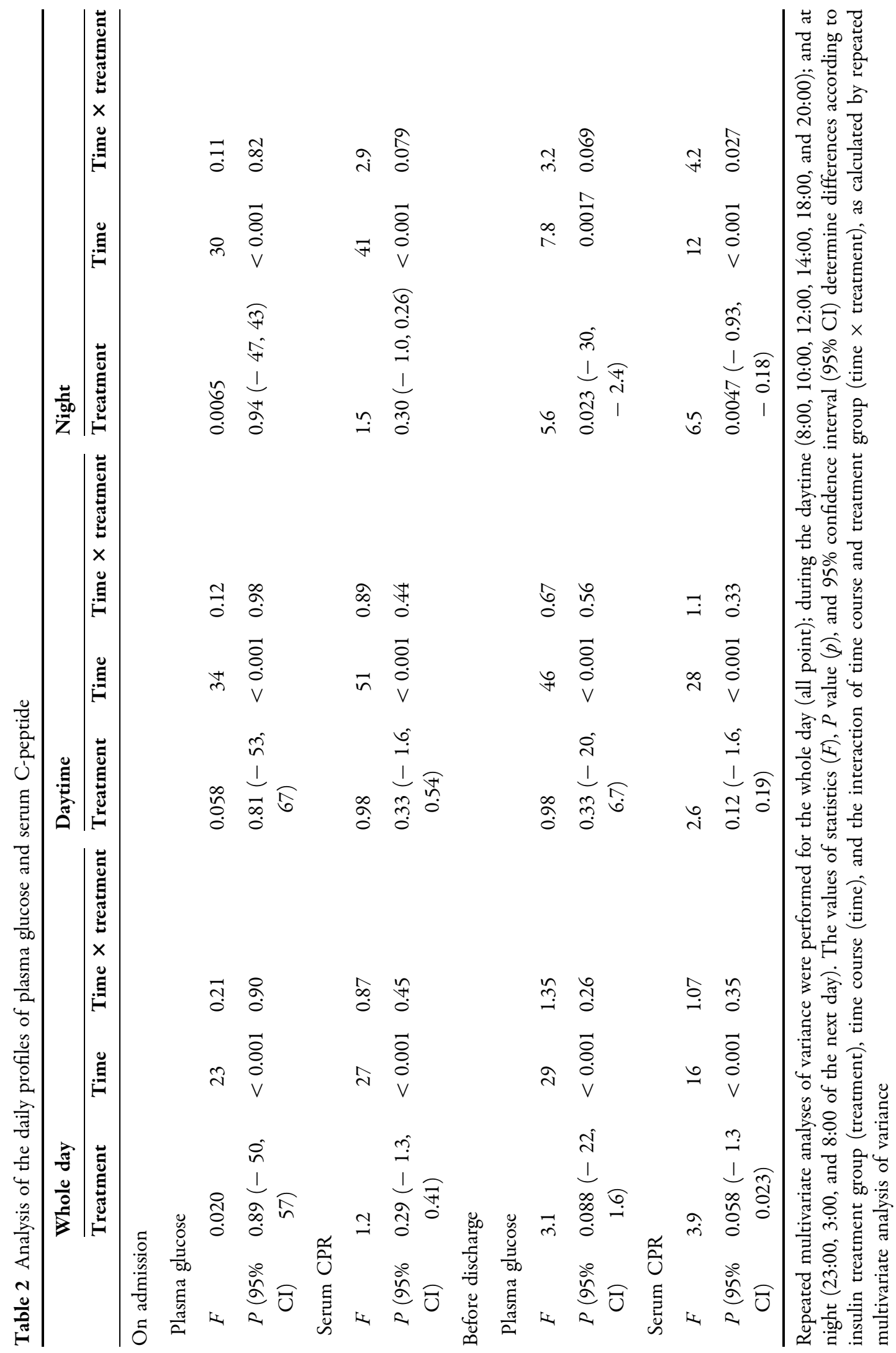




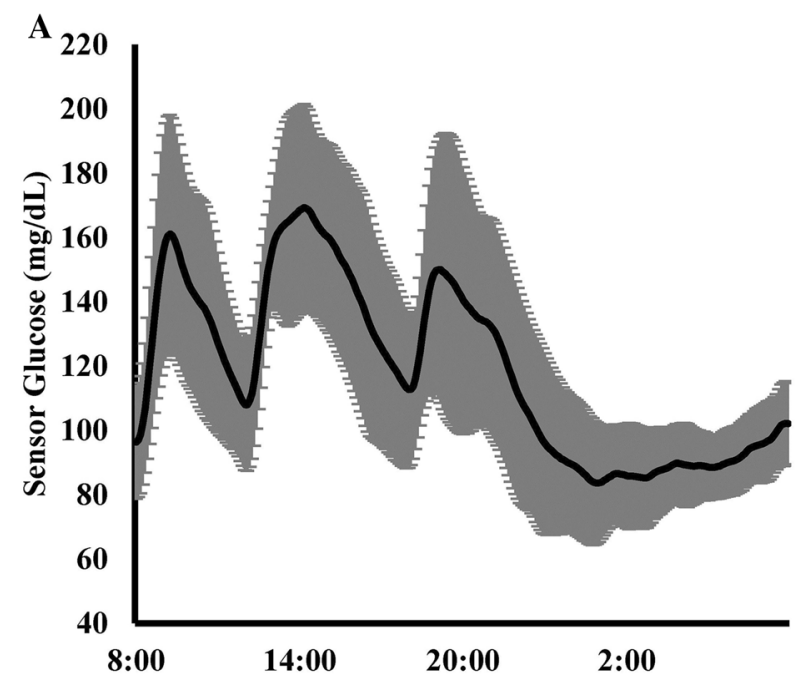

Fig. 3 Continuous glucose monitoring values before discharge. The mean values (solid black line) and the range of standard deviation (gray area) of the continuous glucose

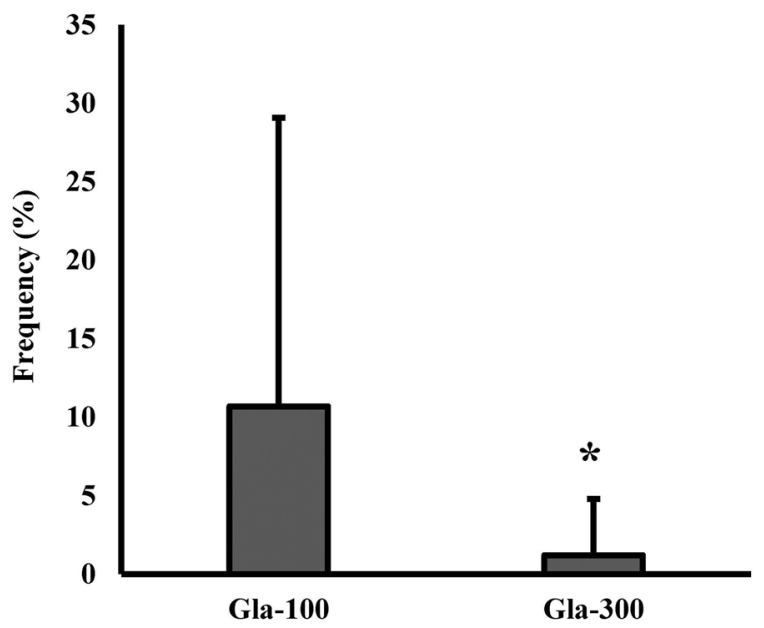

Fig. 4 Frequency of nocturnal hypoglycemia. Data are expressed as mean \pm standard deviation. Gla-100, insulin glargine 100 units $/ \mathrm{mL}$; Gla-300, insulin glargine 300 units/mL. ${ }^{*} P<0.05$ versus the Gla-100 group

phase 3a EDITION programs that compared Gla-300 with Gla-100 [7-12]. The CGM data in this study showed that the nadir of blood glucose profile was around 0:00-1:00 a.m., which is about $4 \mathrm{~h}$ after the injection of basal insulin. In some PK-PD studies, the peak glucose-lowering effect and serum glargine concentration were observed $4 \mathrm{~h}$ after the BBT administration of

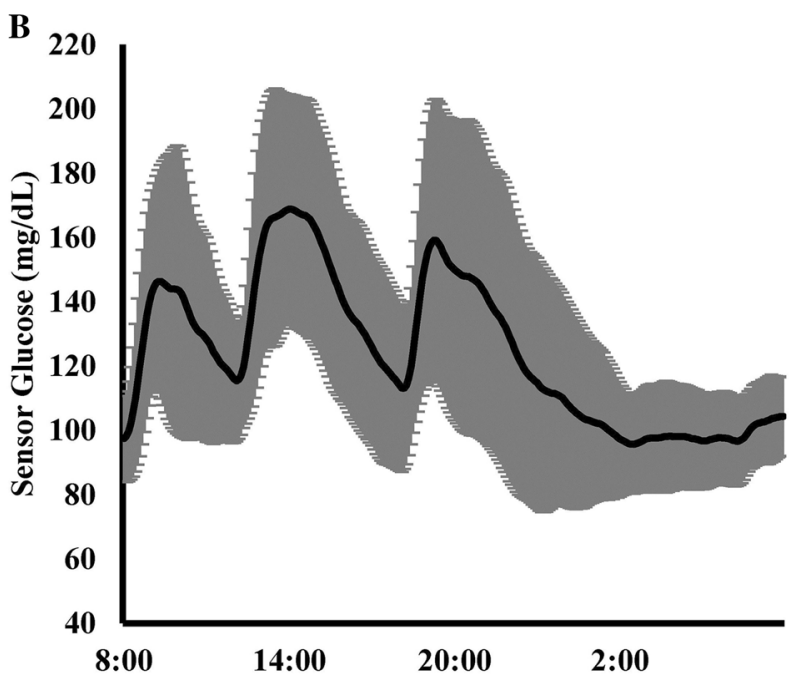

monitoring data before discharge in the a insulin glargine 100 units/mL and $\mathbf{b}$ insulin glargine 300 units/mL groups

both Gla-100 and Gla-300 at 21:00 [4-6]. In the current study, no patients with nocturnal hypoglycemia reported symptoms or showed elevation of fasting plasma glucose, such as that seen in the Somogyi phenomenon [17]. Similar to previous reports, our study suggested that detection of nocturnal hypoglycemia could be challenging without the use of CGM.

Hypoglycemia is known to activate the sympathetic nerves to ameliorate hypoglycemia $[18,19]$; however, this might worsen heart failure. In some clinical trials, hypoglycemia significantly increased the mortality of patients with type 2 diabetes [20, 21]. Administration of beta blocker was reported to reduce the incidence of hospitalization for heart failure [22], whereas intensive glycemic control increased the incidence of cardiovascular deaths [23]. The EMPA-REG OUTCOME study demonstrated that the sodium-glucose cotransporter 2 inhibitor (SGLT2I) reduced the incidence of cardiovascular mortality and hospitalization for heart failure in patients with type 2 diabetes [24]. We previously reported that SGLT2I has a preventive effect on nocturnal hypoglycemia in type 2 diabetic patients on BBT [3]. This preventive effect may have mediated the favorable results for the cardiovascular disease shown in the EMPA-REG OUTCOME study. 
Because of the long PD of insulin degludec, another kind of new basal insulin analogue, a longer time is required to achieve a stable serum concentration of insulin degludec; insulin degludec also takes a long time to stabilize fasting plasma glucose after changing the dose of insulin compared with Gla-100. In the present study, the duration of hospitalization depended on the time to achieve normal glycemic control, regardless of the BBT regimen used. These data suggested that Gla-300 can achieve a stable fasting glucose as fast as Gla100.

This study has some limitations. The unblinded trial and the small sample size might impact the results in this study. Because physical activity during the admission was not assessed, a confounding bias might be introduced. We could not find previous studies about the comparison between Gla-100 and Gla-300 with tightly controlled blood glucose levels and diet therapy in hospitalization; hence, we could not perform a power analysis to determine the sample size. However, although the sample size is small, the preventive effect of Gla-300 on nocturnal hypoglycemia in the present study is similar to the results of previous studies about the comparison between Gla-100 and Gla-300 at an outpatient clinic with a large sample size. Therefore, we believe that the results of the present study are conclusive. Further investigations on the possible complications of nocturnal hypoglycemia are required.

\section{CONCLUSION}

This study documented that Gla-300 had similar efficacy with Gla-100 in controlling blood glucose level, but with less frequent episodes of nocturnal hypoglycemia in type 2 diabetic patients under the tightly controlled diet therapy and careful monitoring of blood glucose in short-term hospitalization.

\section{ACKNOWLEDGEMENTS}

We thank the participants of the study.
Funding. This study and the article processing charges were supported by Grants-in-Aid for Scientific Research from Japan Society for the Promotion of Science (\#17K08936).

Editorial Assistance. The authors would like to thank Enago (www.enago.jp) for the English language review.

Authorship. All named authors meet the International Committee of Medical Journal Editors (ICMJE) criteria for authorship for this article, take responsibility for the integrity of the work as a whole, and have given their approval for this version to be published.

Auhtor Contributions. FO designed and prepared the study, and wrote the manuscript. FO, YN, YY, and YS collected the data. FO and KK analyzed the data. HS and NE reviewed/ edited the manuscript.

Disclosures. Naoya Emoto received research funds from Boehringer Ingelheim Pharmaceuticals, Inc.; Daiichi-Sankyo Co., Ltd.; Dainippon Sumitomo Pharma Co., Ltd.; Kowa Pharmaceutical Co.; Kyowa Hakko Kirin Co.; Mitsubishi Tanabe Pharma Co.; MSD K. K.; Ono Pharmaceutical Co.; Takeda Pharmaceutical Co., Ltd.; and Taisho Toyama Pharmaceutical Co. Naoya Emoto also received lecture fees from Boehringer Ingelheim Pharmaceuticals, Inc.; DaiichiSankyo Co., Ltd.; Kyowa Hakko Kirin Co.; MSD K. K.; Ono Pharmaceutical Co.; and Taisho Toyama Pharmaceutical Co. Hitoshi Sugihara received research funds from Daiichi-Sankyo Co., Ltd.; MSD K. K.; Ono Pharmaceutical Co.; Taisho Toyama Pharmaceutical Co.; Astellas Pharma Inc.; Eli Lilly Japan K. K.; Teijin Pharma Ltd.; and Kaken Pharmaceutical Co., Ltd. Hitoshi Sugihara also received lecture fees from Daiichi-Sankyo Co., Ltd.; Kyowa Hakko Kirin Co.; Mitsubishi Tanabe Pharma Co.; MSD K. K.; Novo Nordisk Inc.; Ono Pharmaceutical Co.; Takeda Pharmaceutical Co., Ltd.; Kaken Pharmaceutical Co., Ltd.; and JCR Pharmaceutical Co., Ltd. Fumitaka Okajima received lecture fees from Astellas Pharma Inc., Astra Zeneca, Boehringer Ingelheim Pharmaceuticals, Inc.; DaiichiSankyo Co., Ltd.; Dainippon Sumitomo Pharma 
Co., Ltd.; Eli Lilly Japan K. K.; Kowa Pharmaceutical Co.; Kyowa Hakko Kirin Co.; Mitsubishi Tanabe Pharma Co.; MSD K. K.; Novartis Pharma K. K.; Novo Nordisk Inc.; Ono Pharmaceutical Co.; Sanofi K.K.; Takeda Pharmaceutical Co., Ltd.; Taisho Toyama Pharmaceutical Co.; and Terumo Co. The funding agencies had no role in research design, the results, or preparation of the manuscript. Yuko Nakamura,Yuji Yamaguchi, Yuki Shuto, and Katsuhito Kato have nothing to disclose.

Compliance with Ethics Guidelines. The study protocol was approved by the ethics committee of Nippon Medical School Chiba Hokusoh Hospital (No.527009) and was registered at UMIN Clinical Trials Registry (UMIN000023360). All procedures followed were in accordance with the ethical standards of the responsible committee on human experimentation (institutional and national) and with the 1964 Declaration of Helsinki, as revised in 2013. Informed consent was obtained from all patients for inclusion in the study.

Data Availability. The datasets generated and/or analyzed during the current study are available from the corresponding author on reasonable request.

Open Access. This article is distributed under the terms of the Creative Commons Attribution-NonCommercial 4.0 International License (http://creativecommons.org/licenses/ by-nc/4.0/), which permits any noncommercial use, distribution, and reproduction in any medium, provided you give appropriate credit to the original author(s) and the source, provide a link to the Creative Commons license, and indicate if changes were made.

\section{REFERENCES}

1. Boland E, Monsod T, Delucia M, et al. Limitations of conventional methods of self-monitoring of blood glucose: lessons learned from 3 days of continuous glucose sensing in pediatric patients with type 1 diabetes. Diabetes Care. 2001;24(11):1858-62.
2. Pieber TR, Eugene-Jolchine I, Derobert E. Efficacy and safety of HOE 901 versus NPH insulin in patients with type 1 diabetes. The European Study Group of HOE 901 in type 1 diabetes. Diabetes Care. 2000;23(2):157-62.

3. Okajima F, Nagamine T, Nakamura $\mathrm{Y}$, et al. Preventive effect of ipragliflozin on nocturnal hypoglycemia in patients with type 2 diabetes treated with basal-bolus insulin therapy: an openlabel, single-center, parallel, randomized control study. J Diabetes Investig. 2017;8(3):341-5.

4. Becker RH, Dahmen R, Bergmann K, et al. New insulin glargine 300 units $\mathrm{L}^{-1}$ provides a more even activity profile and prolonged glycemic control at steady state compared with insulin glargine

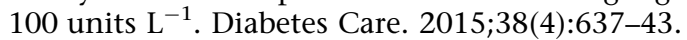

5. Shiramoto $\mathrm{M}$, Eto $\mathrm{T}$, Irie $\mathrm{S}$, et al. Single-dose new insulin glargine $300 \mathrm{U} / \mathrm{ml}$ provides prolonged, stable glycaemic control in Japanese and European people with type 1 diabetes. Diabetes Obes Metab. 2015;17(3):254-60.

6. Steinstraesser A, Schmidt R, Bergmann K, Dahmen $\mathrm{R}$, Becker RH. Investigational new insulin glargine $300 \mathrm{U} / \mathrm{ml}$ has the same metabolism as insulin glargine $100 \mathrm{U} / \mathrm{ml}$. Diabetes Obes Metab. 2014;16(9):873-6.

7. Riddle MC, Bolli GB, Ziemen M, et al. New insulin glargine 300 units $/ \mathrm{mL}$ versus glargine 100 units $/ \mathrm{mL}$ in people with type 2 diabetes using basal and mealtime insulin: glucose control and hypoglycemia in a 6-month randomized controlled trial (EDITION 1). Diabetes Care. 2014;37(10):2755-62.

8. Riddle MC, Yki-Jarvinen H, Bolli GB, et al. One-year sustained glycaemic control and less hypoglycaemia with new insulin glargine $300 \mathrm{U} \mathrm{ml}^{-1}$ compared with $100 \mathrm{U} \mathrm{ml}^{-1}$ in people with type 2 diabetes using basal plus meal-time insulin: the EDITION 1 12-month randomized trial, including 6-month extension. Diabetes Obes Metab. 2015;17(9):835-42.

9. Yki-Jarvinen H, Bergenstal R, Ziemen M, et al. New insulin glargine 300 units/mL versus glargine 100 units/mL in people with type 2 diabetes using oral agents and basal insulin: glucose control and hypoglycemia in a 6-month randomized controlled trial (EDITION 2). Diabetes Care. 2014;37(12):3235-43.

10. Yki-Jarvinen $\mathrm{H}$, Bergenstal RM, Bolli GB, et al. Glycaemic control and hypoglycaemia with new insulin glargine $300 \mathrm{U} / \mathrm{ml}$ versus insulin glargine $100 \mathrm{U} / \mathrm{ml}$ in people with type 2 diabetes using basal insulin and oral antihyperglycaemic drugs: the EDITION 2 randomized 12-month trial including 6-month extension. Diabetes Obes Metab. 2015;17(12):1142-9. 
11. Terauchi Y, Koyama M, Cheng X, et al. New insulin glargine $300 \mathrm{U} / \mathrm{ml}$ versus glargine $100 \mathrm{U} / \mathrm{ml}$ in Japanese people with type 2 diabetes using basal insulin and oral antihyperglycaemic drugs: glucose control and hypoglycaemia in a randomized controlled trial (EDITION JP 2). Diabetes Obes Metab. 2016;18(4):366-74.

12. Bolli GB, Riddle MC, Bergenstal RM, et al. New insulin glargine $300 \mathrm{U} / \mathrm{ml}$ compared with glargine $100 \mathrm{U} / \mathrm{ml}$ in insulin-naive people with type 2 diabetes on oral glucose-lowering drugs: a randomized controlled trial (EDITION 3). Diabetes Obes Metab. 2015;17(4):386-94.

13. Jinnouchi H, Koyama M, Amano A, et al. Continuous glucose monitoring during basal-bolus therapy using insulin glargine $300 \mathrm{U} \mathrm{mL}^{-1}$ and glargine $100 \mathrm{U} \mathrm{mL}^{-1}$ in Japanese people with type 1 diabetes mellitus: a crossover pilot study. Diabetes Ther. 2015;6(2):143-52.

14. Bergenstal RM, Bailey TS, Rodbard D, et al. Comparison of insulin glargine 300 units $/ \mathrm{ml}$ and 100 units/ml in adults with type 1 diabetes: continuous glucose monitoring profiles and variability using morning or evening injections. Diabetes Care. 2017;40(4):554-60.

15. Emoto N, Okajima F, Sugihara H, et al. A socioeconomic and behavioral survey of patients with difficult-to-control type 2 diabetes mellitus reveals an association between diabetic retinopathy and educational attainment. Patient Prefer Adher. 2016;25(10):2151-62.

16. Service FJ, Molnar GD, Rosevear JW, et al. Mean amplitude of glycemic excursions, a measure of diabetic instability. Diabetes. 1970;19(9):644-55.
17. Somogyi M. Exacerbation of diabetes by excess insulin action. Am J Med. 1959;26(2):169-91.

18. Abramson EA, Arky RA, Woeber KA. Effects of propranolol on the hormonal and metabolic responses to insulin-induced hypoglycaemia. Lancet. 1966;2(7478):1386-8.

19. Koivikko ML, Tulppo MP, Kiviniemi AM, et al. Autonomic cardiac regulation during spontaneous nocturnal hypoglycemia in patients with type 1 diabetes. Diabetes Care. 2012;35(7):1585-90.

20. Bonds DE, Miller ME, Bergenstal RM, et al. The association between symptomatic, severe hypoglycaemia and mortality in type 2 diabetes: retrospective epidemiological analysis of the ACCORD study. BMJ. 2010;340:b4909.

21. Zoungas S, Patel A, Chalmers J, et al. Severe hypoglycemia and risks of vascular events and death. N Engl J Med. 2010;363(15):1410-8.

22. Packer M, Bristow MR, Cohn JN, et al. The effect of carvedilol on morbidity and mortality in patients with chronic heart failure. US Carvedilol Heart Failure Study Group. N Engl J Med. 1996;334(21):1349-55.

23. Tsujimoto T, Sugiyama T, Noda M, Kajio H. Intensive glycemic therapy in patients with type 2 diabetes on beta-blockers. Diabetes Care. 2016;39(10):1818-26.

24. Zinman B, Wanner C, Lachin JM, et al. Empagliflozin, cardiovascular outcomes, and mortality in type 2 diabetes. $\mathrm{N}$ Engl J Med. 2015;373(22):2117-28. 